\title{
ESTUDO COMPARATIVO DO RESFRIAMENTO DE LARANJA VALÊNCIA COM AR FORÇADO E COM ÁGUA ${ }^{1}$
}

\author{
Bárbara TERUEL ${ }^{2}$, Luís CORTEZ ${ }^{3}$, Lincoln NEVES FILHO ${ }^{4, *}$
}

\begin{abstract}
RESUMO
Este trabalho apresenta um estudo para comparar o resfriamento rápido de laranja Valência (Citrus sinensis O.), com ar forçado e com água gelada. O sistema de resfriamento rápido com ar forçado operou com um fluxo de ar de $1.933 \mathrm{~m}^{3} / \mathrm{h}(3 \mathrm{l} / \mathrm{s}$ por $\mathrm{kg}$ de produto resfriado), a uma temperatura de $1^{\circ} \mathrm{C}$ e UR=88,4 $\pm 2,0 \%$, com uma velocidade do ar em torno de $1 \mathrm{~m} / \mathrm{s}$. Nos experimentos com água gelada foi utilizado um sistema de imersão, com uma capacidade de $0,23 \mathrm{~m}^{3}$ de água a uma temperatura de aproximadamente $1^{\circ} \mathrm{C}$. Foi determinando o tempo meio e de sete oitavos do resfriamento, partindo das leituras de temperatura, perfazendo-se duas repetições em cada experimento. Os resultados mostraram que, o resfriamento do leito de frutas com ar forçado não é homogêneo, obtendo-se um tempo de sete oitavos do resfriamento que variaram de $107 \mathrm{~min}$ a $170 \mathrm{~min}$, dependendo da posição do fruto no interior da embalagem. Já no sistema com água gelada, o resfriamento acontece uniformemente em todo em todo o leito de frutos, sendo o tempo médio de resfriamento de $57 \mathrm{~min}$.

Palavras-chave: refrigeração; tempo de resfriamento; resfriamento rápido.
\end{abstract}

\section{SUMMARY}

COMPARATIVE STUDY OF COOLING ORANGE WITH FORCED-AIR COOLING AND HYDROCOOLING. This work presents a study to compare the orange Valência (Citrus sinensis O.) cooling with forced-air cooling and hydrocooling. The forced-air cooling system operated with air flow of $1933 \mathrm{~m}^{3} / \mathrm{h}$, at $1^{\circ} \mathrm{C}, \mathrm{RH}=88,4 \pm 2,0 \%$ and air velocity around $1 \mathrm{~m} / \mathrm{s}$. The experiments with immersion hydrocooling systems with capacity of $0,23 \mathrm{~m}^{3}$ of water at $1^{\circ} \mathrm{C}$, approximately. The half-cooling time and seven-eighths cooling time, they are determinate with the temperature data, made two repetitions. The results showed that the cooling the bed fruits, with air cooling, is not homogeneous, and obtained cooling time average, between $107 \mathrm{~min}$ and $170 \mathrm{~min}$, depending on the position the fruit in the package. In the hydrocooling system, the cooling of the bed fruits happens uniformly in the bed, with average cooling time of $57 \mathrm{~min}$.

Keywords: refrigeration; cooling time; pre-cooling.

\section{1 - INTRODUÇÃO}

Após a colheita, o resfriamento rápido, entendido como a rápida remoção do calor de campo dos produtos, deve ser um dos primeiros passos a ser dado. O adequado tratamento pós-colheita, o que inclui o resfriamento, garante várias vantagens como: o consumo de um produto de melhor qualidade, menos perdas para o comerciante, aumento do tempo de comercialização, o que significa um maior beneficio para o agricultor, com menos desperdicio e maiores ganhos [3, 9].

Estima-se que o valor das perdas anuais de produtos frescos, incluindo as frutas, por amadurecimento precoce e por falta de tratamento adequado após a colheita, pode chegar até $50 \%$; dependendo do produto. Entre as várias causas que originam estas perdas estão a não utilização de armazenamento refrigerado após a colheita [6, 10]. Para se evitar esses problemas é necessária a implementação de uma adequada "Cadeia do Frio" $(\mathrm{CF})$, que está definida como o conjunto de sistemas que garantem a manutenção da qualidade dos produtos desde o momento da colheita até o consumo. São elementos fundamentais desta cadeia: as câmaras e sistemas de

1. Recebido para publicação em 27/06/2001. Aceito para publicação em 14/01/2003 (000681).

2. Faculdade de Engenharia Agricola, FEAGRI/UNICAMP. CX. P. 6011 CEP13081-970 - Campinas, SP.E-mail: barbarat@agr.unicamp.br

3. Faculdade de Engenharia Agricola. CX. P. 6120 - CEP 13081-970. E-mail:cortez@agr.unicamp.br

4. Faculdade de Engenharia dos Alimentos. CX. P. 6021-CEP 13081-970. E-mail:neveslin@unicamp.br

* A quem a correspondência deve ser enviada. resfriamento rápido e estocagem, caminhões frigorificados para o transporte terrestre, containers frigorificos para o transporte marítimo, aéreo ou ferroviário, expositores refrigerados de supermercados e geladeiras domésticas e industriais.

Entre os diferentes métodos de resfriamento rápido destacam-se os sistemas de resfriamento com ar forçado (forced-air cooling) e com água gelada (hydrocooling). No primeiro método, os produtos são condicionados no interior de um túnel de ar forçado, estando composto por ventiladores, associados ao sistema de refrigeração da câmara. $\mathrm{O}$ ar resfriado deve entrar em contato com a maior área da embalagem que contêm os produtos, permitindo uma eficiente troca de calor com o meio refrigerado.

Já no segundo método, o calor dos produtos é removido usando-se como meio de resfriamento, água a baixas temperaturas. O resfriamento com água pode ser feito introduzindo os produtos em tanques de imersão, ou, usando-se o método de aspersão, onde a água é aspergida de forma contínua na superficie dos produtos. O resfriamento nestes tipos de sistemas, é rápido e eficiente, podendo ser aplicado numa ampla faixa de produtos [3].

Ambos os sistemas, ar forçado e água, garantem baixos tempos de resfriamento. Mesmo assim, segundo MITCHELL et al. [11], em geral, o sistema de circulação forçada do ar resfria em 1 / 4 a $1 / 10$ em relação ao tempo exigido em câmaras convencionais, mas ainda é duas a três vezes mais lento que o resfriamento com água gelada. A relação entre o tempo e a temperatura é a que caracteriza a eficiência do resfriamento, desta forma 
quanto menor for o tempo de resfriamento, maior será a eficiência do sistema, o que se traduz em produtos que mantém a sua qualidade e uma vida útil de prateleira maior.

Este trabalho foi desenvolvido com o objetivo de se estudar o resfriamento de laranja Valência, utilizando um sistema com ar forçado e um sistema de resfriamento por imersão com água, a fim de comparar as taxas de resfriamento.

\section{2 - MATERIAL E MÉTODOS}

\section{1 - Resfriamento com ar forçado}

Um sistema de ar forçado foi montado no interior de uma câmara de refrigeração (Figura 1). No interior da câmara de resfriamento (A) foi instalado um ventilador centrifugo (2). O ventilador succiona o ar refrigerado que sai do evaporador (1) e o ar resfriado é forçado a passar transversalmente entre o volume de frutos, colocados no túnel de resfriamento (3), o qual está fechado na parte superior e frontal com uma lona (Figura 1a).

(a)

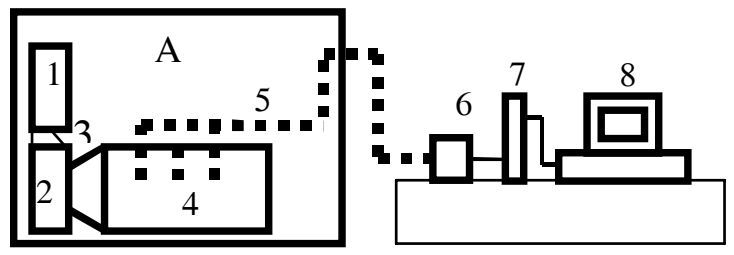

(b)

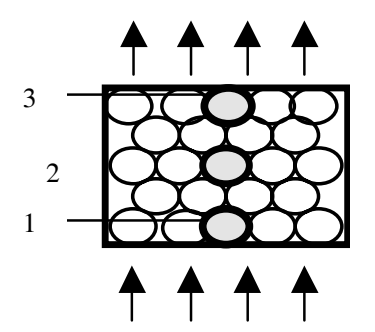

encontra mais afastado da entrada do fluxo de ar, já o ponto 3 coincide com o fruto na posição central da embalagem. Estes frutos encontravam-se coincidindo com a fileira do meio da caixa (Figura 1b). Também foi monitorada a temperatura da superficie em alguns frutos.

A temperatura do ar resfriado e insuflado pelo evaporador foi medida e monitorada, usando-se um termopar colocado no interior da câmara de resfriamento. A temperatura de resfriamento foi de $1^{\circ} \mathrm{C}$, controlada pelo termostato da câmara.

Para o acondicionamento dos frutos utilizou-se uma embalagem plástica $(528 \times 335 \times 310 \mathrm{~mm})$, usada comumente para o transporte dos produtos desde o campo até o galpão embalagem. Estas caixas possuem aproximadamente $60 \%$ de área de abertura, estando disponível para a passagem de ar aproximadamente $40 \%$. Os frutos depois de pesados e medidos (para a determinação do diâmetro), foram colocados no interior da embalagem em arranjo alternado.

A velocidade do fluxo do ar no interior do túnel de ar forçado foi em média de $1,02 \pm 0,15 \mathrm{~m} / \mathrm{s}$. A taxa de ar passando através dos produtos foi de aproximadamente $1.933 \mathrm{~m}^{3} / \mathrm{h}$ (3 1/s x kg produto resfriado).

\section{2 - Resfriamento com água}

Foi utilizado um sistema de resfriamento com água gelada por imersão, mostrado na Figura 2.

O sistema de resfriamento (B) consta de um compressor hermético, um condensador a ar, uma válvula de expansão termostática, um reservatório de líquido e uma bomba.

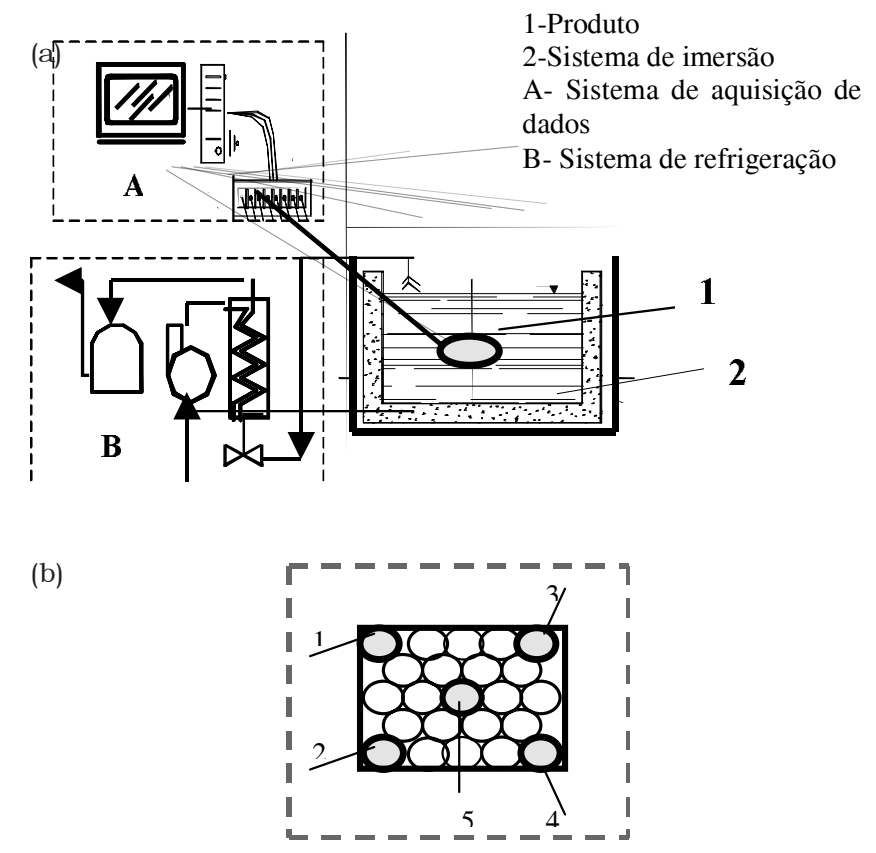

FIGURA 2. a) Elementos que compõem o sistema de resfriamento com água. b) Pontos onde foram inseridos os termopares nos frutos. 
A água que sai do tanque, impulsionada por uma bomba, entra no evaporador, onde se resfria em contato com a serpentina por onde circula o refrigerante, retornando ao tanque de imersão, a uma temperatura mais baixa que a inicial. Assim se fecha o ciclo, até a temperatura da água atingir e ser mantida no ponto fixado no termostato de regulagem, a qual foi de $1^{\circ} \mathrm{C}$.

O tanque de água gelada (780x580x570mm) possui uma capacidade de $0,23 \mathrm{~m}^{3}$ (230L), com uma vazão de aproximadamente $0,001 \mathrm{~m}^{3} / \mathrm{s}$ e isolamento de espuma de vidro com espessura de aproximadamente $5 \mathrm{~cm}$. Uma solução de cloro ativo foi adicionada à água do tanque (aproximadamente 150ppm).

A temperatura dos produtos submetidos ao resfriamento foi medida usando-se termopares T (AWG \# 28), previamente calibrados $\left( \pm 0,3^{\circ} \mathrm{C}\right)$. A temperatura da água no interior do tanque também foi monitorada em dois pontos. O sistema de aquisição de dados por computador (A) foi semelhante ao utilizado nos testes de resfriamento com ar forçado.

Os termopares foram inseridos no centro de 5 laranjas (Figura 2b), dois termopares foram utilizados para medir a temperatura da água de resfriamento, colocando-se um na tubulação de saída da água ao reservatório e outro no interior deste. Um outro termopar foi colocado para medir a temperatura do ar exterior, e outro medindo a temperatura da superficie de alguns frutos.

Uma caixa de laranja (variedade Valência), com aproximadamente $15 \mathrm{~kg}$, foi utilizada para o desenvolvimento dos experimentos. As frutas foram colocadas numa embalagem construida especialmente para o experimento (450x300x300mm), feita com arame em quadrículas, o que garantiu um adequado contato das frutas com a água de resfriamento no tanque.

Antes do início dos experimentos foi feita a determinação do peso e das dimensões dos frutos, escolhendo uma caixa com 80 amostras para a determinação destes parâmetros. Em seguida, os frutos foram acondicionados, no interior da embalagem, em arranjo alternado, em todos os experimentos.

O delineamento experimental adotado foi inteiramente ao acaso (DIC), com duas repetições para cada tratamento aplicado (resfriamento com ar e resfriamento com água). Os valores do tempo de resfriamento, obtidos experimentalmente, foram analisados aplicando uma análise de variância (ANOVA), e o Teste F, para um nivel de significância estatística de 5\% [17].

\section{3 - Determinação do tempo de resfriamento}

Um dos parâmetros utilizados para avaliar a eficiência do resfriamento é a Taxa Adimensional de Temperatura (TAT) [3, 12]. Este parâmetro foi calculado aplicando a equação 1 e utilizando os dados experimentais de temperatura dos frutos.

$T A T=\frac{T c-T a}{T i-T a}$
Onde Tc e Ti são a temperatura no centro e a temperatura inicial das frutas e Ta é a temperatura do meio de resfriamento (ar ou água, dependendo do sistema).

No instante em que TAT for igual a 0,5; o produto terá atingido o tempo de meio resfriamento $\left(\mathrm{TAT}_{1 / 2}\right)$ e quando a TAT for igual a 0,125 os frutos terão atingido o tempo dos sete oitavos do resfriamento $\left(\mathrm{TAT}_{7 / 8}\right)$. As leituras e valores de temperatura estão referidos ao centro dos produtos.

\section{3 - RESULTADOS E DISCUSSÃO}

\section{1 - Resfriamento com ar forçado}

A temperatura inicial média dos frutos foi de 25,2 \pm $0,2^{\circ} \mathrm{C}$, sendo que o tempo de meio resfriamento foi atingido quando a temperatura no centro dos frutos foi de aproximadamente $12,8^{\circ} \mathrm{C}$ e os sete oitavos do tempo de resfriamento quando a temperatura foi da ordem de $3,6^{\circ} \mathrm{C}$. A temperatura média exterior foi de $27,2 \pm 1,3^{\circ} \mathrm{C}$. As frutas apresentaram um peso médio de $216 \pm 12,02 \mathrm{~g}$ e um raio equatorial médio de $3,91 \pm 0,024 \mathrm{~cm}$. A diferença de peso e de diâmetro não foi significativa a um nivel de significância de $5 \%$.

Como pode ser observado na Figura 3, entre os diferentes pontos existe um diferencial da TAT, que se reflete no tempo de resfriamento. A título de exemplo, no instante em que o ponto 1 atinge o tempo meio do resfriamento, no ponto 2 , Tc é de $15,9^{\circ} \mathrm{C}$ e no ponto 3 de $17,5^{\circ} \mathrm{C}$. Estes valores representam uma diferença de $19 \%$ a $27 \%$ ao longo do leito (de $4,7^{\circ} \mathrm{C}$ a $1,6^{\circ} \mathrm{C}$ ). O tempo de meio resfriamento e o desvio padrão para cada ponto no interior do leito são mostrados na Tabela 1.

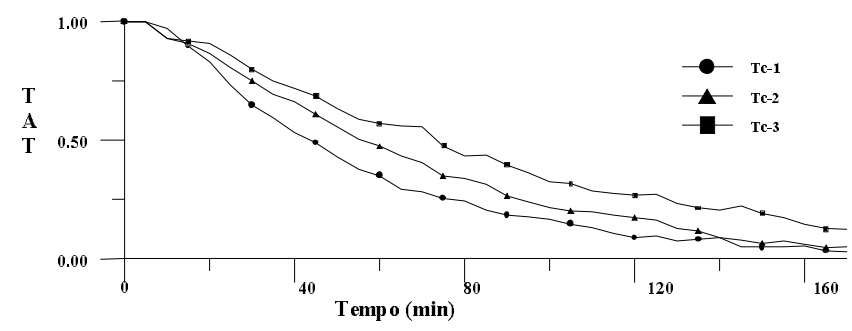

FIGURA 3. Curvas de resfriamento de laranja Valência com ar forçado. Tc- temperatura no centro dos frutos nas diferentes posições no leito.

TABELA 1. Tempo de resfriamento (min) nos pontos monitorados no interior do leito de laranjas resfriadas com ar forçado.

\begin{tabular}{ccc}
\hline \multicolumn{3}{c}{ TAT $_{\mathbf{1} 2}$} \\
\hline Tc-1 & Tc-2 $^{*}$ & Tc-3 \\
\hline $37,5 \pm 2,5^{*}$ & $50,0 \pm 5,0^{*}$ & $67,5 \pm 2,5^{*}$ \\
& T AT $_{7 / 8}$ & \\
$107,5 \pm 7,5^{*}$ & $122,5 \pm 2,5^{*}$ & $170,0 \pm 10,0^{*}$ \\
\hline
\end{tabular}

* Média significativa para um intervalo de confiança de $95 \%$

Os resultados da aplicação da ANOVA e do Teste F mostraram que os valores do tempo de resfriamento fo- 
ram significativamente diferentes nas três posições dentro do leito de frutas, ou seja, dentro da embalagem.

Este diferencial de temperatura pode ter sido provocado pela possivel diminuição do escoamento do ar através dos frutos e o aumento da temperatura do ar de resfriamento. Vários trabalhos da área demonstram a existência de um diferencial de temperatura ao longo do leito $[1,2,4,5,13]$. Porém, os frutos que se encontram em maior contato com o fluxo do ar, resfria-se num tempo menor que aqueles que se encontram numa posição mais afastada $[15,16]$.

Observou-se também a existência de um diferencial de temperatura entre o centro e a temperatura na superficie. $\mathrm{O}$ valor deste $\Delta \mathrm{T}$ oscilou entre, aproximadamente, $1,2^{\circ} \mathrm{C}$ a $10^{\circ} \mathrm{C}$, dependendo da posição do fruto com respeito ao passo do fluxo de ar no leito. A temperatura do ar oscilou na faixa de $1,02 \pm 0,52^{\circ} \mathrm{C}$.

TALBOLT \& CHAU [14], resfriaram morango desde a temperatura ambiente (aproximadamente $28^{\circ} \mathrm{C}$ ) até uma temperatura de $5^{\circ} \mathrm{C}$ e observaram que o tempo dos sete oitavos do resfriamento variou em dependência da taxa de ar e da área de aberturas das embalagens entre $160 \mathrm{~min}$ a $52 \mathrm{~min}$ (taxas de $1,04 \times 10^{-3} \mathrm{~m}^{3} / \mathrm{s}$ à $6,24 \times 10^{-3} \mathrm{~m}^{3} / \mathrm{s}$ ).

\section{2 - Resfriamento com água}

Nos experimentos de resfriamento com água, notouse a existência de homogeneidade no tempo de resfriamento para os diferentes pontos monitorados ( $\mathrm{Ta}$ bela 2). Isto ocorreu por que a caixa de laranja estava submersa no interior do tanque, onde a água de resfriamento encontra-se resfriada e estável, numa faixa de $0,9 \pm 0,7^{\circ} \mathrm{C}$, conseguindo-se um contato eficiente entre o produto e o meio de resfriamento (Figura 4).

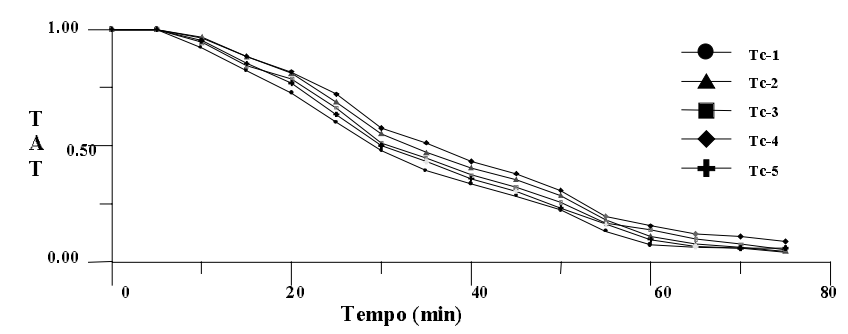

FIGURA 4. Curvas de resfriamento de laranja Valência com água. Tc-temperatura no centro do produto nas diferentes posições no leito.

TABELA 2. Tempo de resfriamento (min) nos pontos monitorados no interior do leito de laranjas resfriadas com água gelada

\begin{tabular}{ccccc}
\hline \multicolumn{5}{c}{ TAT $_{\mathbf{1 / 2}}$} \\
\hline Tc-1 & Tc-2 & Tc-3 & Tc-4 & Tc-5 \\
\hline $22,5 \pm 2,5$ & $22,5 \pm 2,5$ & $25,0 \pm 0,0$ & $27,5 \pm 2,5$ & $25,0 \pm 0,0$ \\
& & TA $_{7 / 8}$ & & \\
$57,5 \pm 2,5$ & $55,00 \pm 5,0$ & $57,5 \pm 2,5$ & $57,5 \pm 2,5$ & $60,0 \pm 5,0$ \\
\hline
\end{tabular}

Os produtos partiram de uma temperatura inicial de $25,7 \pm 1,1^{\circ} \mathrm{C}$, e o tempo meio de resfriamento e o tempo de sete oitavos do resfriamento foram atingidos quando no centro dos produtos a temperatura foi de aproximadamente $12,5^{\circ} \mathrm{C}$ e $3,7^{\circ} \mathrm{C}$, respectivamente. A temperatura do ar exterior oscilou em $26,3 \pm 0,9^{\circ} \mathrm{C}$. Os frutos apresentaram um peso médio de 235, $16 \pm 12,1 \mathrm{~g}$, com um raio equatorial médio de 3,95 $\pm 0,017 \mathrm{~cm}$.

Em alguns pontos, o valor do tempo de resfriamento foi praticamente igual entre as repetições, o que demonstra que foi possivel obter uma adequada reprodutibilidade dos dados. Em média o tempo de resfriamento foi de $24,5 \mathrm{~min}\left(\mathrm{TAT}_{1 / 2}\right.$ ) e de $57,5 \mathrm{~min}\left(\mathrm{TAT}_{7 / 8}\right)$, para todos os frutos da caixa. O desvio padrão médio entre todos os pontos, com relação ao tempo de resfriamento foi de aproximadamente $2 \mathrm{~min}$ (Tabela 2). A análise estatística demonstrou a não existência de diferença significativa entre os valores do tempo de resfriamento obtido nos diferentes pontos.

A análise estatística permitiu aceitar a hipótese de que não existiu diferença entre os valores do tempo de resfriamento obtido nas diferentes posições dentro do leito de frutos contido na embalagem, a um nivel de significância estatística de 5\%.

O monitoramento da temperatura da água permitiu comprovar que as oscilações da temperatura da água foram muito pequenas, o que permite afirmar que o sistema consegue manter a temperatura da água estável, fator este de grande importância para garantir a eficiência do sistema de resfriamento com água.

DINCER \& GENCELI [7] obtiveram tempos de resfriamento da ordem de $27 \mathrm{~min}$ (15kg de pepinos), resfriados com água ( $\mathrm{Tag}=4^{\circ} \mathrm{C}$ ) e um tempo de $47 \mathrm{~min}$, quando resfriados num sistema com ar forçado. Tempos de resfriamento entre $36 \mathrm{~min}$ e $54 \mathrm{~min}$ foram obtidos durante o resfriamento de pêssego, maçã, milho e citricos [3].

GOYETTE et al. [8], determinaram o tempo de resfriamento de talos de brócolis, inicialmente a $24^{\circ} \mathrm{C}$, submetidos a resfriamento com água gelada a uma temperatura de $0^{\circ} \mathrm{C}$. O tempo de resfriamento variou entre $2 \mathrm{~min}$ a $10 \mathrm{~min}$, para talos de $22 \mathrm{~mm}$ a $37 \mathrm{~mm}$ de diâmetro.

\section{3 - Comparação dos resultados obtidos nos siste- mas ar forçado e água}

Na Figura 5 apresentam-se as curvas de resfriamento obtidas durante o resfriamento de laranja Valência em ambos sistemas. Essas curvas correspondem aos valores médios de todos os pontos monitorados nos experimentos.

Observa-se que, efetivamente a diferença do tempo de resfriamento dos frutos entre ambos sistemas é até 3 vezes maior no sistema com ar forçado, se comparado com o sistema de água, como foi discutido por MITCHELL et al. [11]. DINCER \& GENCELI [7] obtiveram tempos quase duas vezes maiores durante o resfriamento de pepino com ar forçado, se comparado com o resfriamento num sistema com água. Em sistemas com água resfriada o coeficiente convectivo de transferência de calor, pode ser até 6 vezes maior se comparado com sistema de resfriamento com ar forçado [3, 15, 16]. 


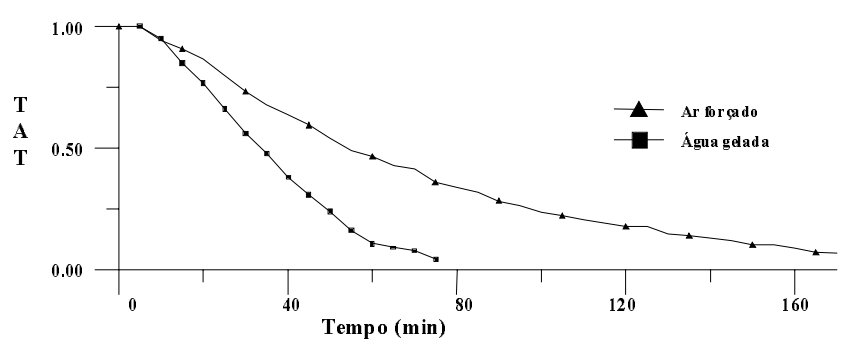

FIGURA 5. Curvas de resfriamento de laranja Valência.

De igual forma, a temperatura da superficie atinge muito mais rapidamente a condição de equilibrio com o meio de resfriamento no sistema com água, se comparado com o sistema de ar forçado. Nos experimentos com ar forçado a diferença entre a temperatura no centro e na superfície chega ser de até $30 \%\left(\right.$ de $7^{\circ} \mathrm{C}$ a $2^{\circ} \mathrm{C}$ de diferença), já nos experimentos com água, a diferença não ultrapassou $1 \%$.

Notou-se também que o tempo de resfriamento no sistema com ar forçado varia em função da posição do fruto no leito, sendo assim o resfriamento do leito não é homogêneo. Nos experimentos com ar forçado este tempo oscilou entre $107 \mathrm{~min}$ a $170 \mathrm{~min}\left(\mathrm{TAT}_{7 / 8}\right)$, uma diferença de quase $70 \mathrm{~min}(37 \%)$ Desta forma as frutas mais próximas da entrada do ar resfriam-se muito mais rapidamente que os frutos mais afastados.

Já nos experimentos com água, a diferença entre os diferentes pontos é da ordem de $3 \mathrm{~min}(5 \%)$, o que demonstra que o resfriamento do leito de frutas é homogêneo, atingindo-se o resfriamento completo num tempo médio de $57,5 \mathrm{~min}$.

Os resultados da ANOVA, assim como da aplicação do Teste $\mathrm{F}$, permitiram comprovar a existência de diferença significativa entre os valores do tempo de resfriamento quando os frutos forma resfriados com ar e quando foram resfriados com água para um intervalo de confiança de $95 \%$.

\section{4 - CONCLUSÕES}

Com este trabalho comprova-se que, desde o ponto de vista da eficiência do resfriamento, o sistema que utiliza água é mais eficiente que o sistema com ar forçado. Sendo assim, quando o produto é apropriado para submetê-lo ao primeiro tipo de resfriamento, recomenda-se o uso de sistemas de resfriamento com água.

Diversos experimentos revelam que este tipo de resfriamento pode ser aplicado numa grande variedade de produtos, dentre eles: maçã, laranja, tangerina, morango, aipo, couve-flor, uvas, espinafre, bananas, entre outros [2, 14].

\section{5 - REFERÊNCIAS}

[1] AlvareZ, G., LetAng, G. Transferts de chaleur et de matiere au sein d' un échangeur de type "palette". Revue du Froid, v. 47, p. 47-49, 1994.

[2] ARIFIN, B. B., CHAU, K. V. Forced air cooling of strawberries. American Society of Agricultural Engineers: ASAE, Paper no. 87-6004, St. Joseph, USA, 1987.

[3] ASHRAE. Systems and Applications. Methods of precooling of fruits, vegetables and flowers. Atlanta: American Society of Heating, Refrigerating and AirConditioning Engineers, p, 1-10, 1994.

[4] BAIRD, C. D., CHAU, K. V., GAFFnEY, J. J. An engineering/economic model for evaluating forcedair cooling systems for fruits and vegetables. Refrigeration Science and Technology. Institute International of Refrigeration, p. 259-266, 1985.

[5] BAIRD, C. D., GAFFney, J. J., TALBOT, M. T. Design criteria for efficient and cost effective forced-air cooling systems for fruits and vegetables. ASHRAE Transactions. v. 94, p. 1434-1453, 1988.

[6] CARRaro, F. A., MANCUSO, C. M. Manual de Exportação de Frutas. Brasília, Ministério da Agricultura, do Abastecimento e da Reforma Agrária, 1994.

[7] DINCER, I., GENCELI, O. F. Cooling process and heat transfer parameters of cylindrical products cooled both in water and air. International Journal Heat Transfer, v. 37, n. 4, p. 625-633, 1994.

[8] GoYetTe, B., Vigneault, C., PANneton, B., RAGHAVAN, G. S. V. Method to evaluate temperature at the surface of horticultural crops. Canadian Agriculture Engineering, v. 38, n. 4, p. 291-295, 1997.

[9] IBRAF. Instituto Brasileiro de Frutas. Boletim Informativo. Relatório, outubro, 1999.

[10] KADER, A.A. Postharvest technology of horticultural crops. 2.ed. Division of Agriculture and Natural Resources. Davis: University of California, n. 3311, 1992. 295p

[11] Mitchell, F. G., Guillou, R., PARsons, R. A. Commercial cooling of fruits and vegetables: Manual Series. N. 43. University of California. 1973. 44 p.

[12] MOHSEnIN, N. N. Thermal properties of foods and agricultural materials. USA. 1980. $405 \mathrm{p}$.

[13] NOVY, M., KIECKBUSH, T.G. Equações para o cálculo do tempo de resfriamento em leitos de frutas esféricas. Ciênc. Tecnol. Aliment., n. 6, p. 1-16, 1986.

[14] TALBOT, M. T., CHAU, K. V. Precooling strawberries. Institute of Food and Agricultural Sciencies. University of Florida. Circular 942. 1991. 8 p

[15] TERUEL, M. B. J. Estudo teórico-experimental do resfriamento com ar forçado de laranja e banana. Campinas, 2000. 300 p. Tese (Doutorado em Planejamento de Sistemas Energéticos). Universidade Estadual de Campinas, Faculdade de Engenharia Mecânica.

[16] TERUEL M. B. J; CORTEZ, L. A. B.; LEAL, P. M.; LIMA, G. A B. Obtenção numérico/experimental das curvas de resfriamento de laranja (variedade Valência). In: Congresso Brasileiro de Engenharia Mecânica (COBEM), 12, 1999.

[17] VIEIRA, S., HOFFMAN, R. Estatística Experimental. São Paulo: Atlas, 1989. 179 p.

\section{6 - AGRADECIMENTOS}

À Fundação de Amparo à Pesquisa do Estado de São Paulo, FAPESP, pelo apoio financeiro à pesquisa. 